\title{
Comparison of 99mTc-DMSA renal scintigraphy with biochemical and histopathological findings in animal models of acute kidney injury
}

\author{
Hossein Fatemikia ${ }^{1} \cdot$ Mohammad Seyedabadi $^{2} \cdot$ Zynab Karimi $^{3} \cdot$ Kiarash Tanha $^{4}$. \\ Majid Assadi ${ }^{2} \cdot K^{2}$ aveh Tanha $^{2}$ (i)
}

Received: 13 February 2017 / Accepted: 25 April 2017

(C) Springer Science+Business Media New York 2017

\begin{abstract}
Biochemical and histological assays are currently used for the diagnosis and characterization of kidney injury. The purpose of this study was to compare technetium-99m-labeled dimercaptosuccinic acid $\left({ }^{99 \mathrm{~m}} \mathrm{Tc}-\right.$ DMSA) renal scintigraphy, as a non-invasive method, with common biochemical and histopathological methods in two animal models of acute kidney injury. Nephrotoxicity was induced either by gentamicin $(100 \mathrm{mg} / \mathrm{kg} /$ day for one week) or unilateral ureteral ligation (UUO). Renal scintigraphy was performed $1 \mathrm{~h}$ after intravenous injection of 99mTc-DMSA ( $3 \mathrm{mCi}$ ). Furthermore, plasma levels of blood urea nitrogen (BUN), creatinine, sodium, and potassium were determined using an autoanalyzer. At the end of experiments, kidneys were excised for the measurement of activity uptake (mCi/gr) using a dose calibrator as well as histopathological examinations with hematoxylin and eosin $(\mathrm{H} \& \mathrm{E})$ staining. There was a significant decrease in 99mTc-DMSA uptake in both gentamicin $(P$ value $=0.049)$ and UUO $(P$ value $=0.034)$ groups, and it was more significant in the former. The levels of BUN and creatinine increased in both gentamicin and UUO groups, while the levels of sodium and potassium remained unchanged. Furthermore, a strong correlation was
\end{abstract}

Kaveh Tanha

k.tanha@bpums.ac.ir

1 Department of Physiology, Medical School, Bushehr University of Medical Sciences, Bushehr, Iran

2 The Persian Gulf Nuclear Medicine Research Center, Bushehr University of Medical Sciences, Bushehr, Iran

3 Shiraz Nephron Urology Research Center, Shiraz University of Medical Sciences, Shiraz, Iran

4 Department of Biostatistics, School of Public Health, Iran University of Medical Sciences, Tehran, Iran found between DMSA uptake and histopathological findings. Scintigraphy with $99 \mathrm{mTc}-\mathrm{DMSA}$ is capable of detection of kidney injury in both gentamicin and UUO groups. Moreover, a significant correlation was found between scintigraphy parameters and histopathological findings. This suggests $99 \mathrm{mTc}-\mathrm{DMSA}$ as a non-invasive method for the evaluation of kidney injury induced by drugs or anatomical disorders.

Keywords Small-animal imaging · Renal scintigraphy · 99mTc-DMSA · Renal function · Gentamicin ·

Nephrotoxicity

\section{Introduction}

Acute kidney injury (AKI), also known as acute renal failure is characterized by the rapid loss of kidney function followed by attenuated urine output and the accumulation of waste products such as nitrogen metabolites. Treatment is mainly supportive because no specific treatment has emerged to decrease acute kidney injury or enhance recovery. However, patients with AKI are at higher risk for the development of chronic kidney disease. Therefore, early diagnosis of AKI will help prevent the development of chronic conditions with higher rates of morbidity and mortality [1]. In this regard, several experimental models are used for understanding the pathophysiology of kidney injury. Likewise, these methods are implemented for the development of reliable methods for accurate diagnosis and potential treatments $[2,3]$. These include animal models derived from drug-induced nephrotoxicity (gentamicin or cisplatin), nephrotoxicity with ureteral obstruction, and renal ischemic/reperfusion studies $[4,5]$.

Kidney injury is associated with accumulated levels of waste materials as well as alteration of histopathological 
parameters. Therefore, biochemical markers such as blood urea nitrogen $(\mathrm{BUN})$ and serum creatinine $(\mathrm{Cr})$ along with histological examinations are used for the evaluation of kidney function [6, 7]. However, biochemical assays lack sensitivity to relay acute injury. This is proven by the fact that any changes in the levels of BUN and $\mathrm{Cr}$ require the loss of at least $75 \%$ of functional nephrons [8-10]. Hence, there should be an extensive damage to the kidneys to cause an alteration in the levels of BUN and Cr. Also, in spite of high accuracy, histological examinations have some disadvantages. These include long run-time, difficult interpretation, difficulties in following up studies, and the invasive nature of the methods [11]. Therefore, development of non-invasive methods with high accuracy and sensitivity is of interest for the early diagnosis of renal dysfunction.

Non-invasive radionuclide imaging has been used for the assessment of relative renal function [12, 13]. In particular, renal functional properties can be evaluated using 99mTc-labeled compounds, such as mercaptoacetyltriglycine (MAG3), diethylenetriamine pentaacetic acid (DTPA), and dimercaptosuccinic acid (DMSA) $[11,14,15]$. In this regard, 99mTc-DMSA is considered as the radiotracer of choice for the assessment of cortical damage, estimating regional renal function and detection of renal parenchymal abnormalities owing to its localization in proximal tubules [16-20]. In addition, 99mTc-DMSA uptake is found to correlate with renal blood flow (RBF), glomerular filtration rate (GFR), and creatinine clearance [21-23]. However, it is yet to be clarified whether $99 \mathrm{mTc}-$ DMSA scintigraphy can relay acute kidney damage resulting from anatomical ligation or pharmacological interventions. In fact, if the results of DMSA scan correlate well with biochemical and histological findings, this scan can be used in pre-clinical studies for the screening of renoprotective agents.

The purpose of this study was to compare $99 \mathrm{mTc}$ DMSA renal scintigraphy with common biochemical and histopathological methods in two animal models of acute kidney injury; (1) gentamicin-induced nephrotoxicity and (2) complete unilateral ureteral obstruction in rats.

\section{Materials and methods}

\section{Animals and experimental groups}

Male Sprague-Dawley rats (250-300 g) were used in the present study. Rats were kept three per cage in a temperature-controlled room $\left(23 \pm 1{ }^{\circ} \mathrm{C}\right)$ with $12 \mathrm{~h} \mathrm{light/dark}$ cycle and had free access to standard rodent diet and water. The rats were randomly divided into four groups $(n=8)$ : gentamicin (GM), gentamicin sham (GMS), unilateral ureteral obstruction (UUO), and unilateral ureteral obstruction sham (UUOS) groups. All protocols conformed to the guidelines for the care and handling of animals prepared by the Ethics Committee in accordance with the international conventions on animal experimentation [24].

\section{Induction of nephrotoxicity}

Nephrotoxicity was induced either by gentamicin or unilateral ureteral ligation. The first group was treated with gentamicin $(100 \mathrm{mg} / \mathrm{kg} / \mathrm{day}$, i.p.) in a volume of $1 \mathrm{ml}$ for 7 days $[25,26]$. Rats in the sham group received saline of the same volume as the former group.

Nephrotoxicity was induced via unilateral ureteral ligation in the second group. The rats were anesthetized (ketamine $(70 \mathrm{mg} / \mathrm{kg}$, i.p.) and xylazine $(10 \mathrm{mg} / \mathrm{kg}$, i.p.) and placed on a thermoregulated surgical table to keep body temperature at $37 \pm 1{ }^{\circ} \mathrm{C}$. After disinfection with Povidone Iodine, a midline incision was made, and the left ureter was identified. The left ureter was then ligated using a 4-0 silk string at two points and was cut between the ligatures in order to prevent retrograde urinary tract infection. Finally, the incision was closed in 2 layers with 4-0 silk strings [27-29]. The left ureter of rats in the sham group (UUOS) was exposed and manipulated simply without ligation. Animals were allowed to recover from anesthesia prior to returning to individual cages.

\section{Renal scintigraphy and image analysis}

Renal scintigraphy was accomplished 7 days after drug or surgical induction of nephrotoxicity under general anesthesia using HiReSPECT (Parto Negar Persia Co., Iran), a dedicated small-animal SPECT system [30, 31]. The imaging system is equipped with two gamma camera detector heads fitted with parallel-hole collimators and $\mathrm{CsI}(\mathrm{Na})$ crystal with $38 \times 80$ pixels $(1 \times 1 \times 5 \mathrm{~mm})$. Radionuclide scintigraphy was performed after the preparation of $99 \mathrm{mTc}$-DMSA according to the manufacturer's recommendations. According to the results of our previous study [32], the recommended image acquisition time for renal scintigraphy with $99 \mathrm{mTc}$-DMSA in the rat is $1-2 \mathrm{~h}$ post-injection. Hence, $1 \mathrm{~h}$ after injection of $3 \mathrm{mCi}$ of $99 \mathrm{mTc}$-DMSA through the tail vein, two opposed planar images (anterior and posterior) were collected in a $38 \times 80$ matrix.

Regions of interest (ROIs) were selected around the kidneys and background areas for both anterior and posterior images. After background correction, the geometric mean of the anterior and posterior images was used for the calculation of renal uptake. DMSA uptake was determined for the left kidney by the calculation of the photon counts per pixel and the relative uptake which was calculated as 
the left kidney counts to the sum of the left and right kidney counts.

\section{Biochemical assays}

After radionuclide scan, blood samples were taken by heart puncture and centrifuged at $5000 \mathrm{rpm}$ for $10 \mathrm{~min}$. The resulting plasma was stored at $-20{ }^{\circ} \mathrm{C}$ for the determination of BUN, creatinine, sodium $\left(\mathrm{Na}^{+}\right)$, and potassium $\left(\mathrm{K}^{+}\right)$levels. The serum levels of BUN and creatinine were determined using an autoanalyzer. Likewise, the serum concentrations of $\mathrm{Na}^{+}$and $\mathrm{K}^{+}$ions were determined using the EasyLyte analyzer.

\section{Histopathological examination}

At the end of the experiments, the abdomen of the animals was opened through the midline, renal pedicles were tied off, and then kidneys were excised. The activity uptake of the kidneys was measured using a dose calibrator (CRC-25R, Capintec, Inc.). The kidneys were then preserved in $10 \%$ formaldehyde buffer for histological studies.

The preserved kidneys were embedded in paraffin and $5 \mu \mathrm{m}$ sections were obtained by a microtome. Sections were then subjected to routine staining with hematoxylin and eosin (H\&E). Consequently, a pathologist colleague was asked to examine each section in at least 10 randomly selected non-overlapping fields under a light microscope in a blind manner. The renal histopathology was quantified for the degree of Bowman's space enlargement, tubular cell necrosis, exfoliated cells in the lumen, vascular congestion, and intratubular casts [33, 34]. The level of each manifestation was graded according to the changes involved in comparison with the control group; scoring 0 for no changes, 1 for changes less than 20\%, 2 for $20-40 \%$ of change, 3 for $40-60 \%$ of change, 4 for $60-80 \%$ of change, and 5 for greater than $80 \%$ of change compared to the control. Finally, the total histopathological score was calculated as the sum of scores for all regions of the nephrons.

\section{Statistical analysis}

The Statistical Package for Social Sciences (SPSS), version 24, (SPSS Inc., Chicago, IL, USA) was used for statistical analysis and a $P$ value of less than 0.05 was considered statistically significant. Continuous variables are presented as mean \pm SD. Mann-Whitney tests were used to compare the variables between the GM and GMS and also between the UUO and UUOS groups.

\section{Results}

\section{Radionuclide scan}

The background activity increased in both GM and UUO groups compared to their sham groups, particularly in the GM group (Fig. 1). The ratio of the relative uptake in the left kidneys of the GM and UUO groups to their amounts in their sham groups were 1 and 0.5 , respectively (Table 1). The count per pixel decreased from 212 in saline treated to 84 in gentamicin-treated rats. Likewise, this parameter decreased from 190 in UUO sham to 78 UUO group. The ratios of the count per pixel in both of the models to their sham groups (GM/GMS and UUO/UUOS) were equal to 0.4.

\section{Plasma variables}

The BUN level significantly increased from 20 to $72 \mathrm{mg} / \mathrm{dl}$ $(P$ value $=0.001)$ and 24.5 to $31 \mathrm{mg} / \mathrm{dl}(P$ value $=0.02)$ in the GM and UUO groups, respectively. The incensement of the BUN in the GM group was 3 times more than that in the UUO group. The serum levels of creatinine produced a similar pattern. Indeed, the creatinine level increased from 0.5 to $2.16 \mathrm{mg} / \mathrm{dl}(P$ value $<0.001)$ in the GM group and from 0.53 to $0.65 \mathrm{mg} / \mathrm{dl}$ ( $P$ value $<0.05)$ in the UUO group. On the other hand, there were no significant changes in the sodium and potassium levels in either of the nephrotoxicity models compared to their sham groups.

\section{Renal histopathology}

The tubular and vascular structures of cortex in right kidney of UUO group were similar to that in the UUOS group which showed a normal appearance (not shown). In contrast, the cortex area of the GM groups and the left kidney of the UUO groups showed enlargement of Bowman space as well as epithelial cell damage in proximal tubule (PT) and thick ascending limb (TAL) of loop of Henle (Fig. 2). Likewise, the epithelial cells of PT and TAL were destroyed at the cortex of the GM group and the left kidney of the UUO group.

Table 2 shows the quantification of the renal histopathology. Total scores in GM and UUO groups were 4.76 and 1.72, respectively. Injuries in each nephrotoxic group were higher than their corresponding sham group. Furthermore, kidney damage in GM group was more severe than that observed in the left kidney of the UUO group.

\section{Discussion}

A variety of pharmacological agents or pathophysiological conditions can induce kidney damage. In particular, the clinical application of aminoglycosides is limited because 
Fig. 1 Renal scintigraphy images of the animals. The 99mTc-DMSA uptake decreased in both kidneys of the rat in GM group and also in the left kidney of the rat in the UUO group. Background activity increased for the rats in both of the GM and the UUO groups. $G M$ gentamicin-induced nephrotoxicity, GMS GM sham operation, $U U O$ unilateral ureteral obstruction, UUOS UUO sham operation
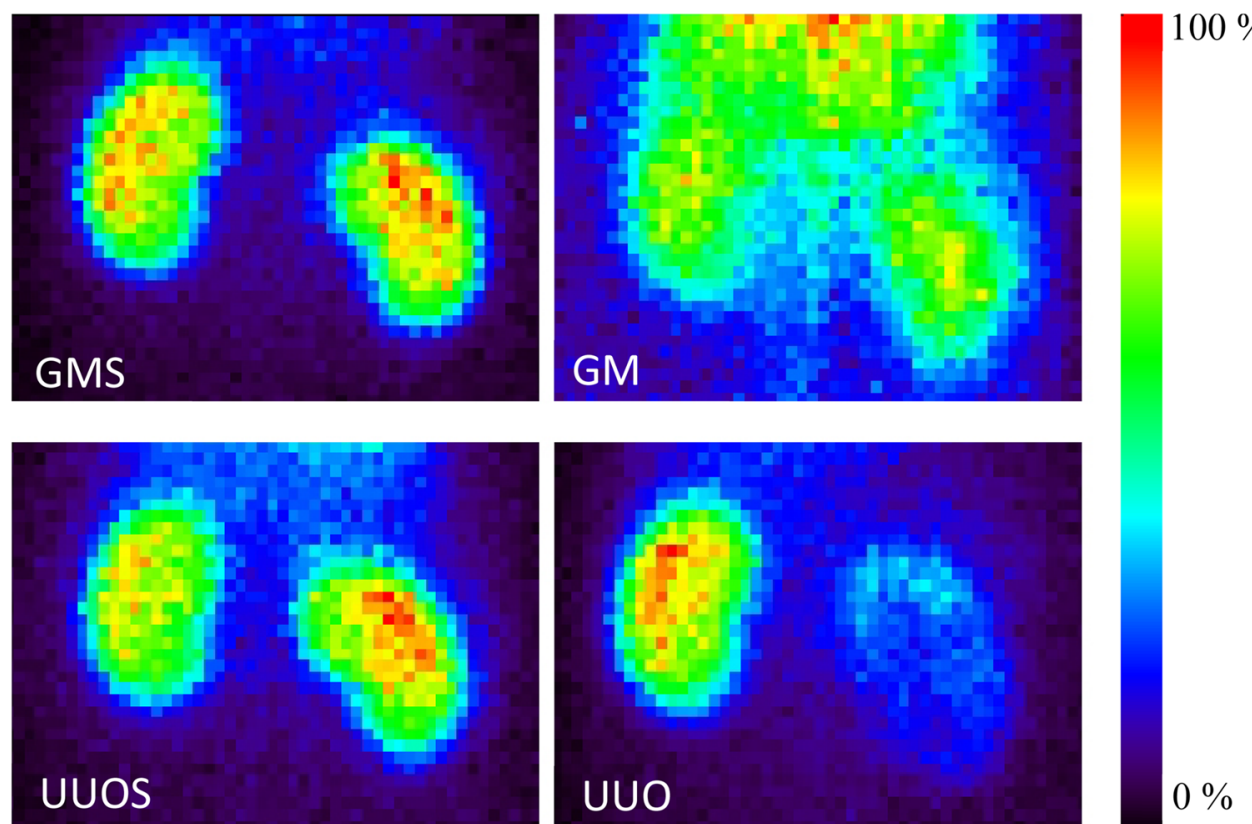

Table 1 Biochemical analysis of plasma levels of BUN, creatinine, sodium, and potassium and results of tissue Tc-99m DMSA uptake in the GM, GMS, UUO, and UUOS groups

\begin{tabular}{llllllll}
\hline Groups & $\begin{array}{l}\text { Activity } \\
(\mathrm{mCi}) / \mathrm{gr}\end{array}$ & $\begin{array}{l}\text { Tc-99m DMSA Relative uptake } \\
{[\mathrm{LC} /(\mathrm{RC}+\mathrm{LC})] \%}\end{array}$ & $\begin{array}{l}\text { Tc-99m DMSA } \\
\text { uptake }(\mathrm{Count} / \mathrm{pixel})\end{array}$ & BUN (mg/dl) & $\begin{array}{l}\mathrm{Creatinine} \\
(\mathrm{mg} / \mathrm{dl})\end{array}$ & $\begin{array}{l}\mathrm{Na}^{+}(\mu \mathrm{mol} / \\
\mathrm{ml})\end{array}$ & $\begin{array}{l}\mathrm{K}^{+}(\mu \mathrm{mol} / \\
\mathrm{ml})\end{array}$ \\
\hline GMS & $0.45 \pm 0.06$ & $48.8 \pm 0.69$ & $212.66 \pm 15.04$ & $20.33 \pm 5.13$ & $0.51 \pm 0.04$ & $143 \pm 1.0$ & $3.83 \pm 0.32$ \\
GM & $0.087 \pm 0.01$ & $47.06 \pm 4.3$ & $84.33 \pm 5.5$ & $72 \pm 1.41$ & $2.16 \pm 0.12$ & $138 \pm 2.82$ & $3.1 \pm 0.56$ \\
$P$ value & 0.046 & $\mathrm{NS}$ & 0.049 & 0.001 & $<0.001$ & $\mathrm{NS}$ & $\mathrm{NS}$ \\
UUOS & $0.42 \pm 0.04$ & $52.33 \pm 1.94$ & $190.33 \pm 7.57$ & $24.5 \pm 0.70$ & $0.53 \pm 0.00$ & $142.5 \pm 2.12$ & $4.08 \pm 0.02$ \\
UUO & $0.137 \pm 0.06$ & $28.36 \pm 5.24$ & $78.25 \pm 14.75$ & $31 \pm 2.54$ & $0.65 \pm 0.06$ & $143.6 \pm 2.07$ & $3.98 \pm 0.60$ \\
$P$ value & 0.032 & 0.034 & 0.034 & 0.020 & 0.044 & $\mathrm{NS}$ & $\mathrm{NS}$ \\
\hline
\end{tabular}

$G M$ gentamicin-induced nephrotoxicity, GMS GM sham operation, $U U O$ unilateral ureteral obstruction, $U U O S$ UUO sham operation, $L C$ left kidney count, $R C$ right kidney count, $B U N$ blood urea nitrogen, $N a$ sodium, $K$ potassium, $N S$ not significant

Results were expressed as mean \pm standard deviation

of nephrotoxicity and ototoxicity of these agents. In fact, aminoglycosides contain charged amine functional groups and are basic in normal body $\mathrm{pH}$. Hence, they exhibit potent interaction with acidic phospholipids of the plasma membrane. This suggests acidic phospholipids as the first binding site for aminoglycosides. Moreover, Megaline, a multiligand endocytic receptor, also serves as the binding site for these agents [35]. Consequently, they undergo endocytosis and concentrate in lysosomes, the Golgi apparatus, and endoplasmic reticulum. Once a threshold is reached, they accumulate in the cytosol, alter mitochondrial function, and induce apoptosis and necrosis. Furthermore, they hamper the functions of several transporters compromising electrolyte balance and cell viability. They also decrease glomerular filtration and renal blood flow through the increase of intracellular calcium levels, production of vasoconstrictors such as thromboxane A2 and endothelin 1, activation of the renin-angiotensin-aldosterone system, and induction of platelet-activating factor [36]. In addition, aminoglycosides enhance the formation of free radicals and lipid peroxidation in the presence of iron salts [37].

Biochemical and histological assays are currently used for the diagnosis and characterization of kidney injury in small-animal experiments. However, these methods either are invasive and time-consuming or lack the desirable sensitivity for early diagnosis. Therefore, non-invasive methods are of interest for the evaluation of acute kidney injury. In this study, we evaluated the quantitative $99 \mathrm{mTc}-$ DMSA renal scintigraphy in comparison with biochemical and histopathological assays in two animal models of acute kidney injury; nephrotoxicity was induced by either gentamicin or unilateral ureteral obstruction. In particular, DMSA uptake in the kidneys was studied in terms of 3 
Fig. 2 Renal tissue damages after nephrotoxicity by gentamicin and the left kidney of UUO groups. Representative light microphotographs of the juxtamedullary cortex of rats. Hematoxylin and eosin (H\&E) staining, with indications of black arrows to Bowman's space, white arrows to tubular damage

Table 2 Scores of tubular cell injury in cortex area of the left kidneys in the experimental groups of rats
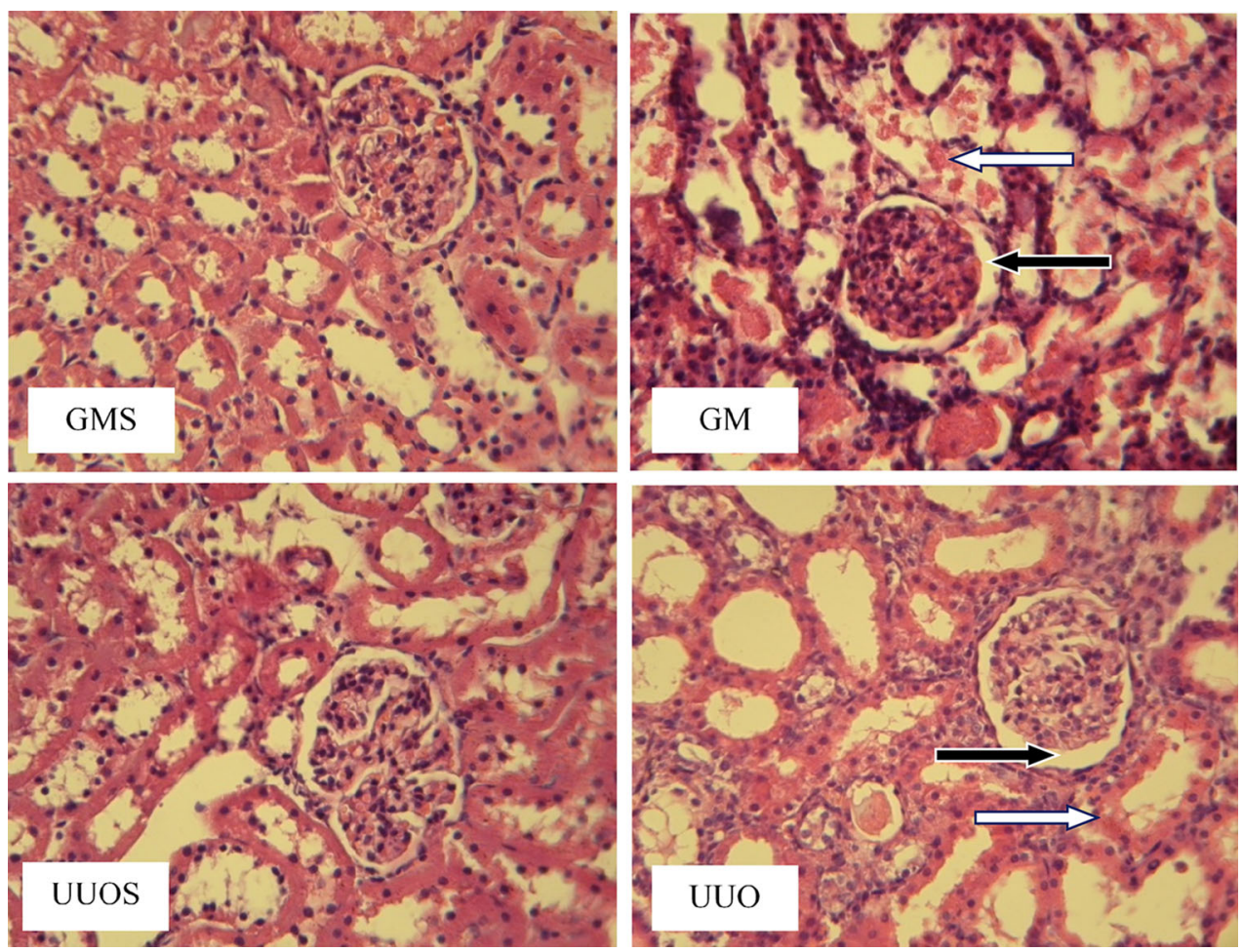

\begin{tabular}{lllll}
\hline Variables & GMS & GM & UUOS & UUO \\
\hline Bowman's space enlargement & 0.00 & $1.07 \pm 0.22$ & 0.00 & $0.87 \pm 0.04$ \\
Proximal tubule injury & 0.00 & $1.73 \pm 0.3$ & 0.00 & $0.67 \pm 0.08$ \\
Thick ascending limb injury & 0.00 & $1.96 \pm 0.03$ & 0.00 & $0.17 \pm 0.08$ \\
Distal tubule injury & 0.00 & 0.00 & 0.00 & 0.00 \\
Collecting duct injury & 0.00 & 0.00 & 0.00 & 0.00 \\
Total histopathological score & 0.00 & $4.76 \pm 0.23$ & 0.00 & $1.72 \pm 0.25$
\end{tabular}

parameters. The first parameter was activity per gram which earned by direct measurement of the concentrated activity in the kidney using a dose calibrator. Furthermore, kidney counts per pixel and renal relative uptake were also determined via quantitative assessment of scintigraphy images. The activity per gram and count per pixel describe renal uptake in the unit of kidney mass or pixel of the kidney in images, respectively.

There was a significant reduction in 99mTc-DMSA uptake in nephrotoxic groups. This is shown as lower activity per gram of kidney (dosimetry) as well as lower count/pixel (scintigraphy) in GM and UUO rats compared to the corresponding sham groups. In addition, gentamycin caused more reduction in these values than did ureteral ligation (Table 1). In this regard, our results are in concordance with Cabuk et al. [15]. They investigated the potential renoprotective effect of erdosteine against gentamicin-induced renal dysfunction using Tc99m-DMSA scintigraphy in rats. They showed a significant decrement in $99 \mathrm{mTc}$-DMSA uptake in GM group compared to the controls. Also, our results were in agreement with those of Yamada et al. in this case [21]. They evaluated the 99mTcDMSA renal uptake as an absolute renal function by comparison with endogenous creatinine clearance in cisplatin-induced nephrotoxicity. They showed that $99 \mathrm{mTc}-$ DMSA uptake in the treated group after 7 days of treatment was significantly lower than that of the control group.

The relative renal uptake describes the ratio of DMSA uptake in the left kidney to the total uptake in both kidneys. As indicated in Table 1, relative renal uptake was similar between gentamicin and its control group. This could be explained by the fact that gentamicin causes loss of function in both kidneys. Therefore, relative renal uptake will be similar in spite of a significant reduction in total kidney uptake. The results of this study are comparable to those of Lora-Michiels et al. [38]. They studied the efficiency of 99mTc-DTPA, 99mTc-MAG 3 , and 99mTc-DMSA in gentamicin-induced renal injury in mongrel dogs. They 
showed that renal function was constant in comparison to each other and the relative renal function values did not change for different doses of gentamicin. On the other hand, our results showed that UUO caused a significant decrease both in total kidney uptake and relative renal uptake. This is explained by the fact that ureteral ligation was performed only in the left kidney while the right ureter remained intact.

Background activity in nephrotoxic rats was higher than the corresponding sham groups. Moreover, the difference between gentamicin and its control was much higher than that between UUO and its sham-operated group. This could be due to the compensation of the renal function from the intact right kidney in UUO group. In other words, the uptake of DMSA by intact kidney decreases background activity. Such an effect is absent in gentamicin-treated rats because of the involvement of both kidneys in this model. The results of this experiment are comparable to those of Lora-Michiels et al. [38]. They showed that after the establishment of injury, the definition of the kidney was poor due to incensement of the overall background activity.

Nephrotoxicity significantly increased the serum levels of BUN and creatinine in both models. In addition, such an increase was much higher in gentamicin model compared to ureteral ligation. In this regard, Cabuk et al. [15], also, reported a significant increase of BUN and creatinine in gentamicin-treated rats. This is while Lora-Michiels et al. indicated that there was no significant correlation between BUN or creatinine levels and gentamicin injected dose [38]. This implies that BUN and creatinine could not indicate the severity of kidney damage [39]. We induced nephrotoxicity with a fixed dose of gentamicin. Hence, we could not investigate whether or not there is a relationship between the gentamicin dose and renal uptake of DMSA. In this regard, serum levels of sodium (Na) and potassium $(\mathrm{K})$ changed neither in gentamycin nor in ureteral ligation model. The latter could be due to the compensatory effects of the intact right kidney. However, the exact mechanisms behind this observation in the former group require further investigation.

There were some histopathological changes in the left renal cortex in both models of nephrotoxicity. This includes Bowman's space enlargement, tubular cell necrosis, exfoliated cells in the lumen, vascular congestion, intratubular casts, and reduction of brush borders. Furthermore, the total histopathological score was much higher in gentamicin-treated rats compared to UUO group $(4.76 \pm 0.23$ vs. $1.72 \pm 0.25)$.

The kidney damage in gentamycin-treated rats was more severe than in UUO group. This is evident both in scintigraphy and in biochemical or histopathological assays. Also, there was a good agreement between the Tc99m-DMSA uptake measured by the dose calibrator and the Tc99m-DMSA counts measured by the gamma camera. This shows the ability of $99 \mathrm{mTc}$-DMSA scan to determine the amount of kidney damage using quantitative image analysis. Our findings showed that there was a good correlation between the results of histopathological and scintigraphy assays which was supported by dose calibrator results. This highlights the fact that quantitative assessment of DMSA uptake can be implemented not only for the detection of kidney injury but also for the analysis of the extent of the injury. The results were in agreement with previous studies of evaluation hyper-functional uptake of 99mTc-DMSA with contralateral kidney due to unilateral kidney dysfunction $[11,40]$.

\section{Conclusion}

Quantitative renal 99mTc-DMSA scintigraphy offers a sensitive and reliable non-invasive method for the detection of kidney damage in two animal models of nephrotoxicity. In addition, renal scintigraphy has the capacity to quantify not only damage to the kidneys but also the extent of the damage.

Acknowledgements This work was financially supported by Grant No. 9228 to K. Tanha from the deputy of research of Bushehr University of Medical Sciences.

\section{Compliance with ethical standards}

Conflict of interest The authors declare that they have no competing interests.

\section{References}

1. Bellomo R, Kellum JA, Ronco C (2012) Acute kidney injury. Lancet 380(9843):756-766

2. Lieberthal W, Nigam SK (2000) Acute renal failure. II. Experimental models of acute renal failure: imperfect but indispensable. Am J Physiol Renal Physiol 278(1):F1-F12

3. Ramesh G, Ranganathan P (2014) Mouse models and methods for studying human disease, acute kidney injury (AKI). In: Mouse Genetics. Springer, New York, pp 421-436

4. Dai C, Kiss LP, Liu Y (2008) Animal models of kidney diseases. In: Sourcebook of models for biomedical research. Springer, Berlin, pp 657-664

5. Singh AP, Muthuraman A, Jaggi AS, Singh N, Grover K, Dhawan R (2012) Animal models of acute renal failure. Pharmacol Rep 64(1):31-44

6. Najafi H, Owji SM, Kamali-Sarvestani E, Moosavi SMS (2016) A1-Adenosine receptor activation has biphasic roles in development of acute kidney injury at 4 and $24 \mathrm{~h}$ of reperfusion following ischaemia in rats. Exp Physiol 101(7):913-931

7. Nuransoy A, Beytur A, Polat A, Samdanci E, Sagir M, Parlakpinar H (2015) Protective effect of sitagliptin against renal ischemia reperfusion injury in rats. Ren Fail 37(4):687-693

8. Edelstein CL (2008) Biomarkers of acute kidney injury. Adv Chronic Kidney Dis 15(3):222-234 
9. Krawiec D, Badertscher R 2nd, Twardock A, Rubin S, Gelberg H (1986) Evaluation of 99mTc-diethylenetriaminepentaacetic acid nuclear imaging for quantitative determination of the glomerular filtration rate of dogs. Am J Vet Res 47(10):2175-2179

10. Labato M, Ross L (1991) Plasma disappearance of creatinine as a renal function test in the dog. Res Vet Sci 50(3):253-258

11. Kwak W, Jang H-S, Belay T, Kim J, Ha YS, Lee SW, Ahn B-C, Lee J, Park KM, Yoo J (2011) Evaluation of kidney repair capacity using 99m Tc-DMSA in ischemia/reperfusion injury models. Biochem Biophys Res Commun 406(1):7-12

12. Silva-Rodríguez J, Cortés J, Pardo-Montero J, Pérez-Fentes D, Herranz M, Ruibal Á, Aguiar P (2015) In vivo quantification of renal function in mice using clinical gamma cameras. Phys Med 31(3):242-247

13. Melis M, de Swart J, de Visser M, Berndsen SC, Koelewijn S, Valkema R, Boerman OC, Krenning EP, de Jong M (2010) Dynamic and static small-animal SPECT in rats for monitoring renal function after $177 \mathrm{Lu}$-labeled Tyr3-octreotate radionuclide therapy. J Nucl Med 51(12):1962-1968

14. Lee WG, Kim J-H, Kim JM, Shim KM, Kang SS, Chae HI, Choi SH (2010) Renal uptakes of 99mTc-MAG3, 99mTc-DTPA, and 99mTc-DMSA in rabbits with unilateral ureteral obstruction. Vivo 24(2):137-139

15. Cabuk M, Gurel A, Sen F, Demircan N (2008) Renoprotective effect of erdosteine in rats against gentamicin nephrotoxicity: a comparison of 99mTc-DMSA uptake with biochemical studies. Mol Cell Biochem 308(1-2):35-42

16. Weyer K, Nielsen R, Petersen SV, Christensen EI, Rehling M, Birn H (2013) Renal uptake of 99mTc-dimercaptosuccinic acid is dependent on normal proximal tubule receptor-mediated endocytosis. J Nucl Med 54(1):159-165

17. Daniel GB, Mitchell SK, Mawby D, Sackman JE, Schmidt D (1999) Renal nuclear medicine: a review. Vet Radiol Ultrasound 40(6):572-587

18. Yürekli Y, Ünak P, Yenisey Ç, Ertay T, Müftüler FZB, Medine Eİ (2011) L-carnitine protection against cisplatin nephrotoxicity in rats: comparison with amifostin using quantitative renal Tc 99m DMSA uptake. Mol Imaging Radionucl Ther 20(1):1

19. Pérez-Fentes D, Cortés J, Gude F, García C, Ruibal Á, Aguiar P (2014) Does percutaneous nephrolithotomy and its outcomes have an impact on renal function? Quantitative analysis using SPECT-CT DMSA. Urolithiasis 42(5):461-467

20. Aguiar P, Perez-Fentes D, Garrido M, García C, Ruibal A, Cortes J (2016) A method for estimating DMSA SPECT renal function for assessing the effect of percutaneous nephrolithotripsy on the treated pole. Q J Nucl Med Mol Imaging 60(2):154-162

21. Yamada M (1991) Assessment of 99mTc-DMSA renography and uptake compared with creatinine clearance in rats with drug-induced nephrotoxicity-II. Cisplatin-induced nephrotoxicity. Kaku Igaku 28(4):347-354

22. Kawamura J, Hosokawa S, Yoshida O (1979) Renal function studies using 99mTc-dimercaptosuccinic acid. Clin Nucl Med 4(1):39-46

23. Taylor A (1982) Quantitation of renal function with static imaging agents. Semin Nucl Med 4:330-344

24. Kolar R (2006) Animal experimentation. Sci Eng Ethics 12(1):111-122

25. Baradaran A, Rafieian-kopaei M (2013) Histopathological study of the combination of metformin and garlic juice for the attenuation of gentamicin renal toxicity in rats. J Renal Inj Prev 2(1):15-21

26. Tavafi M, Ahmadvand H, Tamjidipour A, Rasolian B (2014) Effect of normobaric hyperoxia on gentamicin-induced nephrotoxicity in rats. Iran J Basic Med Sci 17(4):287

27. Wu M, Wen M, Chiu Y, Chiou Y, Shu K, Tang M-J (2006) Rapamycin attenuates unilateral ureteral obstruction-induced renal fibrosis. Kidney Int 69(11):2029-2036

28. Tantawy MN, Jiang R, Wang F, Takahashi K, Peterson TE, Zemel D, Hao C-M, Fujita H, Harris RC, Quarles CC (2012) Assessment of renal function in mice with unilateral ureteral obstruction using 99m Tc-MAG3 dynamic scintigraphy. BMC Nephrol 13(1): 1

29. Maeshima A, Mishima K, Yamashita S, Nakasatomi M, Miya M, Sakurai N, Sakairi T, Ikeuchi H, Hiromura K, Hasegawa Y (2014) Follistatin, an activin antagonist, ameliorates renal interstitial fibrosis in a rat model of unilateral ureteral obstruction. BioMed Res Int 2014:1-10

30. Pashazadeh A, Tanha K, Jafarian-Dehkordi F, Assadi M, Moji V, Zeraatkar N, Ay M (2015) Experimental evaluation of the performance of HiReSPECT scanner: a high-resolution SPECT system for small animal imaging. Front Biomed Technol 1(3):222-227

31. Moji V, Zeratkar N, Farahani MH, Aghamiri MR, Sajedi S, Teimourian B, Ghafarian P, Sarkar S, Ay MR (2014) Performance evaluation of a newly developed high-resolution, dualhead animal SPECT system based on the NEMA NU1-2007 standard. J Appl Clin Med Phys 15(6):267-278

32. Tanha K, Fatemikia H, Assadi M, Seyedabadi M (2017) Assessment of the maximum uptake time of 99mTc-DMSA in renal scintigraphy in rat. Iran J Nucl Med (in press)

33. Moosavi S, Bayat G, Owji S, Panjehshahin M (2009) Early renal post-ischaemic tissue damage and dysfunction with contribution of A1-adenosine receptor activation in rat. Nephrology 14(2):179-188

34. Karimi Z, Ketabchi F, Alebrahimdehkordi N, Fatemikia H, Owji SM, Moosavi SMS (2016) Renal ischemia/reperfusion against nephrectomy for induction of acute lung injury in rats. Ren Fail 38(9):1503-1515

35. Nagai J, Takano M (2004) Molecular aspects of renal handling of aminoglycosides and strategies for preventing the nephrotoxicity. Drug Metab Pharmacokinet 19(3):159-170

36. Wargo KA, Edwards JD (2014) Aminoglycoside-induced nephrotoxicity. J Pharm Pract 27(6):573-577

37. Priuska EM, Schacht J (1995) Formation of free radicals by gentamicin and iron and evidence for an iron/gentamicin complex. Biochem Pharmacol 50(11):1749-1752

38. Lora-Michiels M, Anzola K, Amaya G, Solano M (2001) Quantitative and qualitative scintigraphic measurement of renal function in dogs exposed to toxic doses of Gentamicin. Vet Radiol Ultrasound 42(6):553-561

39. Finco D, Tabaru H, Brown S, Barsanti J (1993) Endogenous creatinine clearance measurement of glomerular filtration rate in dogs. Am J Vet Res 54(10):1575-1578

40. Yen TC, Chen WP, Chang SL, Liu RS, Yeh SH, Lin CY (1996) Technetium-99m-DMSA renal SPECT in diagnosing and monitoring pediatric acute pyelonephritis. J Nucl Med 37(8):1349-1353 